\title{
Continuous Neural Plasticity in the Olfactory Intrabulbar Circuitry
}

\author{
Diana M. Cummings and Leonardo Belluscio \\ Developmental Neural Plasticity Unit, National Institute of Neurological Disorders and Stroke, National Institutes of Health, Bethesda, Maryland 20892
}

In the mammalian brain each olfactory bulb contains two mirror-symmetric glomerular maps linked through a set of reciprocal intrabulbar projections. These projections connect isofunctional odor columns through synapses in the internal plexiform layer (IPL) to produce an intrabulbar map. Developmental studies show that initially intrabulbar projections broadly target the IPL on the opposite side of the bulb and refine postnatally to their adult precision by 7 weeks of age in an activity-dependent manner (Marks et al., 2006). In this study, we sought to determine the capacity of intrabulbar map to recover its precision after disruption. Using reversible naris closure in both juvenile and adult mice, we distorted the intrabulbar map and then removed the blocks for varying survival periods. Our results reveal that returning normal olfactory experience can indeed drive the re-refinement of intrabulbar projections but requires 9 weeks. Since activity also affects olfactory sensory neurons (OSNs) (Suh et al., 2006), we further examined the consequence of activity deprivation on P2-expressing OSNs and their associated glomeruli. Our findings indicate that while naris closure caused a marked decrease in P2-OSN number and P2-glomerular volume, axonal convergence was not lost and both were quickly restored within 3 weeks. By contrast, synaptic contacts within the IPL also decreased with sensory deprivation but required at least 6 weeks to recover. Thus, we conclude that recovery of the glomerular map precedes and likely drives the refinement of the intrabulbar map while IPL contacts recover gradually, possibly setting the pace for intrabulbar circuit restoration.

\section{Introduction}

The mammalian olfactory system detects chemical information from the external environment and translates that information into neural signals that are conveyed directly to the brain. In the nasal cavity, each olfactory sensory neuron (OSN) expresses one of $\sim 1300$ odorant receptors (ORs) (Buck and Axel, 1991) and responds to a distinct set of odorants (Zhuang and Matsunami, 2007). OSNs expressing the same receptor are generally found scattered within one of four epithelial zones and project axons ipsilaterally, usually to a stereotypic pair of glomeruli (Ressler et al., 1994; Vassar et al., 1994; Mombaerts et al., 1996). This convergence of OSN axons produces a mirror-symmetric organization of isofunctional glomerular pairs on the surface of the olfactory bulb (OB) known as the "glomerular map." Each isofunctional pair of glomeruli is further linked through a set of intrabulbar projections mediated by superficial tufted cells (STCs) positioned just beneath the glomerular layer. STCs extend axons that terminate on granule cell dendrites in the inner plexiform layer (IPL) on the opposite side of the same OB (Liu and Shipley, 1994; Belluscio et al., 2002; Lodovichi et al., 2003). These precise reciprocal projections form an "intrabulbar map" that may allow the two

\footnotetext{
Received April 3, 2010; revised May 10, 2010; accepted May 18, 2010.

This work was supported by the Intramural Research Program of the National Institutes of Health-National Institute of Neurological Disorders and Stroke.

Correspondence should be addressed to Dr. Leonardo Belluscio, Developmental Neural Plasticity Unit, National Institute of Neurological Disorders and Stroke, Porter Neuroscience Research Center, Building 35, Room 3A-116, 35 Convent Drive, MSC 3703, Bethesda, MD 20892-3703.E-mail: bellusc1@ninds.nih.gov.

DOI:10.1523/JNEUROSCI.1717-10.2010

Copyright $\odot 2010$ the authors $\quad 0270-6474 / 10 / 309172-09 \$ 15.00 / 0$
}

halves of the $\mathrm{OB}$ to coordinate responses to odorants (for review, see Cummings and Belluscio, 2008).

Recent studies have shown that the glomerular and intrabulbar maps achieve specificity during development, and that both can be influenced by odorant-induced activity. Enhancing odorant-induced activity with odorant conditioning during development has been shown to accelerate the refinement of both glomerular (Kerr and Belluscio, 2006) and intrabulbar circuitry (Marks et al., 2006). Long-term naris closure causes altered glomerular refinement if it begins soon after birth (Nakatani et al., 2003; Zou et al., 2004), but not when initiated later in life. Interestingly, the intrabulbar map appears to be extremely responsive to reductions in afferent activity since olfactory deprivation beginning either during development or adulthood results in a broadening of intrabulbar projections (Marks et al., 2006). Thus, while the axonal projections that make up the glomerular and intrabulbar maps both change with altered levels of olfactory stimulation, they do not exhibit the same degree of plasticity.

Responses to naris closure have been well characterized and include alterations in epithelial thickness, neuronal survival and death, OB size, and biochemistry (for review, see Brunjes, 1994). Since the olfactory system continuously incorporates new neurons into its circuitry and exhibits a tremendous capacity for reorganization, we sought to investigate its potential to recover from disruption caused by a temporary reduction in odorantinduced activity. Prior work has shown that reinstating normal levels of afferent activity following naris closure leads to recovery of OB size through neuronal replacement (Cummings et al., 1997); however, little is known about the olfactory system's capacity to reestablish the specificity of its axonal projections. Here 
we investigated whether intrabulbar projections can regain their accuracy following naris occlusion either before or after the intrabulbar map had matured. Furthermore, we examined this intrabulbar projection plasticity with respect to the coincident changes that occur in the olfactory epithelium $(\mathrm{OE})$, the glomerular map, and the density of postsynaptic contacts in the internal plexiform layer of the $\mathrm{OB}$.

\section{Materials and Methods}

Mice. Experiments were performed on C57/B6 mice and on two transgenic mouse lines: rI7 $\rightarrow$ M71 mice (Bozza et al., 2002) and P2-IREStaulacZ mice (Mombaerts et al., 1996). All experimental subjects were male mice. All animal procedures were in compliance with the National Institutes of Health guidelines and approved by the National Institute of Neurological Disorders and Stroke/Animal Care and Use Committee.

Reversible naris closure. $\mathrm{rI} 7 \rightarrow \mathrm{M} 71$ and P2-IRES-taulacZ mice underwent reversible naris closure from 4 to 7 or 7 to 10 weeks of age using small plugs as described previously (Cummings and Brunjes, 1997; Cummings et al., 1997). Plugs were constructed out of polyethylene (PE) tubing (PE50, Becton Dickinson), silk surgical suture thread (size 3-0), and filaments from unwaxed dental floss. Mice were briefly anesthetized using an inhalant anesthetic, the exterior surface of the plug was lightly coated with a sterile lubricant (Puralube Ointment, Fougera), and plugs were gently inserted into the external naris of each mouse such that only $2 \mathrm{~mm}$ of floss extended from the external naris. After 3 weeks of unilateral naris closure, plugs were removed by lightly anesthetizing each mouse and gently pulling on the floss with forceps.

Neural tracer injections. Targeted injections of fluorescent dextrantetramethylrhodamine (Invitrogen) were done as described previously (Lodovichi et al., 2003; Marks et al., 2006). Briefly, mice were anesthetized with ketamine $(200 \mathrm{mg} / \mathrm{kg}) /$ xylazine $(10 \mathrm{mg} / \mathrm{kg})$ and maintained with halothane $\left(1-3 \%\right.$ in $\left.100 \% \mathrm{O}_{2}\right)$. Animals were then placed in a stereotaxic apparatus, the scalp was resected, and a small portion of the skull over each olfactory bulb was removed. A fluorescent dye [10\% dextrantetramethylrhodamine (TMR), 3000 molecular weight, Invitrogen] was iontophoretically injected $(+10 \mu \mathrm{A}, 200 \mathrm{~ms}$ duration, $2500 \mathrm{~ms}$ interval, 120 pulses for $5 \mathrm{~min}$ ) into a glomerulus through a quartz micropipette (7-10 $\mu \mathrm{m}$ tip diameter). At 12-24 h after injection, mice were killed with an overdose of $200 \mathrm{mg} / \mathrm{kg}$ ketamine and transcardially perfused with $1 \times$ PBS followed by 4\% PFA. Brains were removed and postfixed, cryoprotected in $30 \%$ sucrose, sectioned on a freezing microtome (60 $\mu \mathrm{m}$ horizontal sections), and mounted onto slides using Vectashield mounting medium containing 4',6'-diamidino-2-phenylindole (DAPI; Vector Laboratories).

Image acquisition and quantification. Images of tracer injection and projection sites were collected using a Zeiss LSM 510 laser scanning confocal microscope at both $10 \times$ and $20 \times$ magnifications (excitation 554 , emission 580). The diameter of the projection site was defined as the longest distance between branch points of labeled axons using a line drawn parallel to the mitral cell layer. Ratios were calculated between the diameters of the injection site and corresponding projection site, averaged, and reported \pm SEM. Comparisons of averaged projection: injection site ratios were analyzed with a one-way ANOVA followed by post hoc comparisons using the Holm-Sidak method.

Epithelial tissue processing and quantification. P2-IRES-taulacZ mice that underwent reversible naris closure from 7 to 10 weeks of age were whole-mount dissected to expose intact turbinates and X-gal stained as described previously (Cummings et al., 2000). P2-LacZ-positive neurons along turbinate II were counted and average cell counts within the experimental and control sides of the nasal cavity were compared using the Student's $t$ test.

$O B$ tissue processing and glomerular volume quantification. The OBs of P2-IRES-taulac $Z$ mice were postfixed, cryoprotected, and sectioned (40 $\mu \mathrm{m})$ before immunohistochemical staining for $\beta$-galactosidase ( $\beta$-gal; Promega; antibody dilution $=1: 1000)$ using a standard procedure for immunofluorescence (Cummings et al., 2000). Sections were mounted onto slides in serial order and coverslipped using Vectashield mounting medium containing DAPI (Vector Laboratories). Confocal images of lateral and medial $\beta$-gal-ir glomeruli were collected using a Zeiss LSM 510 laser scanning confocal microscope at $40 \times$ magnification (excitation 496, emission 519), and glomerular volumes measured using a stereological method (Brunjes et al., 1985; Cummings et al., 1997). Briefly, each glomerular volume was calculated by (1) measuring the area of each $\beta$-gal-ir glomerulus in every third $1.5 \mu \mathrm{m}$ optical section using Zeiss LSM software, (2) computing the average volume between adjacent measured optical sections, and (3) summing those volumes and adding the volume of the glomerulus in the first and last sections in which it appeared. Comparisons of averaged lateral and medial glomerular volumes within experimental and control OBs were done using the Student's $t$ test.

Reconstruction of axonal tufts. Three-dimensional (3D) reconstructions of axonal tufts ( $n=3$ mice per group) were performed on raw data confocal stacks using Imaris software (Bitplane). Reconstructions were manually examined and edited to ensure reconstruction reliability. Quantitative parameters included the total axonal filament length and the number of branch points. Branch point density, i.e., the number of branch points/filament length, was compared across groups of mice using a one-way ANOVA followed by the Holm-Sidak post hoc test.

Immunofluorescence and image analysis. Mice that underwent naris closure from 7 to 10 weeks followed by 0,3 , or 6 weeks of reopening, and 10 -week-old control mice were perfused transcardially with $1 \times$ PBS followed by $4 \%$ PFA. The OBs were postfixed, cryoprotected, sectioned ( 40 $\mu \mathrm{m}$ ) coronally, and processed for immunofluorescence for synaptoporin (rabbit anti-synaptoporin; Synaptic Systems; antibody dilution = 1:1000) using a standard procedure for immunohistochemistry (Cummings et al., 2000) with an anti-rabbit secondary conjugated to Cy3 (Jackson ImmunoResearch). Sections were mounted serially onto slides and coverslipped using Vectashield mounting medium (Vector Laboratories). Confocal images of synaptoporin-ir were collected with a Zeiss LSM 510 laser scanning confocal microscope (excitation 543, emission $570)$ using a $40 \times, 0.9 \mathrm{NA}$ oil objective. For each group ( $n=3$ mice/ group), a $z$-series was collected from each olfactory bulb at $\sim 30 \%$ and $50 \%$ of the anterior and posterior extent of the OB on the medial side. Five-micrometer $z$-stacks encompassing both the IPL and external plexiform layer (EPL) were collected at $1 \mu \mathrm{m}$ intervals as 8 bit, $1024 \times 1024$ images. For each bulb synaptoporin puncta density was quantified from single images located halfway through each $z$-stack using Volocity Image Analysis Software (PerkinElmer). Images were first normalized (0-255), then synaptoporin-positive puncta were counted based on intensity thresholding (2 SDs above the mean) and size (minimal size: $0.095 \mu \mathrm{m}^{2}$ ). These threshold levels were kept constant across all measured sections. Puncta counts were measured in the IPL and EPL by manually outlining each layer of interest in every optical section using the DAPI counterstain to delineate laminar boundaries. Counts of synaptoporin-ir puncta were divided by corresponding laminar area calculations to determine density values. Data were analyzed using a one-way ANOVA, followed by the Holm-Sidak post hoc test.

\section{Results}

\section{Intrabulbar map recovery following naris closure}

To determine the capacity of the intrabulbar map to recover following a reduction in afferent activity, we performed reversible naris closure from either 4-7 or 7-10 weeks of age in mice and then assessed the specificity of their intrabulbar projections at 0 , 3 , 6, or 9 weeks after removal of the naris block. At each time point, localized tracer injections of $10 \%$ TMR were performed and the tissue was processed for histology and analysis $(n=4-6$ in all groups) (see supplemental Table 1, available at www. jneurosci.org as supplemental material, for numbers of mice/ group, average injection, and projection measurements and ratios). In mice that underwent reversible naris closure from 4 to 7 weeks, projection to injection measurements showed that the broad intrabulbar projection patterns observed after 3 weeks of olfactory deprivation (Fig. $1 B, H$ ) gradually return to control levels by 9 weeks (Fig. $1 E, K$ ), suggesting that STC projection specificity does recover (Fig. 1Y). Data were analyzed using a 
one-way ANOVA that revealed significant differences across groups for both the $4-7$ and $7-10$ week blocks $\left(F_{(7,28)}=52.78, p<\right.$ 0.001 and $F_{(7,29)}=58.69, p<0.001$, respectively), yet no significant differences were found across control groups. Post hoc tests that compared the mean projection to injection ratios of age-matched experimental and control groups using the Holm-Sidak method showed significant differences after naris closure from 4 to 7 weeks $\left({ }^{*} p<0.001 ; t=15.40\right)$, and after naris closure followed by 3 weeks of recovery $\left.{ }^{\star} p<0.001 ; t=6.29\right)$ or 6 weeks of recovery $\left({ }^{*} p<0.001 ; t=4.34\right)$, but not in naris-closed animals followed by 9 weeks of recovery $(p=0.44 ; t=0.78$ ) (Fig. $1 Z$ ).

Similarly, post hoc comparisons showed that naris closure that began after the intrabulbar map had already matured, from 7 to 10 weeks, also resulted in a significant broadening of the projections as compared to age-matched controls $\left({ }^{\star} p<\right.$ $0.001 ; t=12.04$ ) (Fig. $1 N, T)$. Significant differences in intrabulbar projections were observed after deprivation from 7 to 10 weeks followed by 3 weeks $\left({ }^{*} p<0.001\right.$; $t=10.23)$ or 6 weeks $\left({ }^{\star} p<0.001 ; t=\right.$ $3.90)$ of normal odorant activity compared to age-matched controls. However, no significant difference was found after naris closure and 9 weeks of recovery $(p=$ $0.16 ; t=1.44$ ) (Fig. 1Q, $W, Z$ ). Thus, olfactory deprivation that begins either before or after the intrabulbar map is mature causes projections to broaden significantly. In both age groups, reopening the naris leads to a gradual re-refinement of intrabulbar projections that takes between 6 and 9 weeks (Fig. $1 Y, Z$ ).

To verify that the observed changes in intrabulbar projection refinement are not unique to $\mathrm{rI7} \rightarrow$ M71 transgenic mice, we also performed injections using C57/B6 mice, the background strain for both $\mathrm{rI7} \rightarrow \mathrm{M} 71$ and P2-IRES-taulacZ transgenic mice. C57/B6 mice underwent naris closure from 7 to 10 weeks followed by targeted tracer injections at 0,3 , or 6 weeks after block removal (see supplemental Fig. 1, available at www.jneurosci.org as supplemental material). The resulting projection to injection ratios in C57/B6 mice were virtually identical to those observed following injections of $\mathrm{rI7} \rightarrow \mathrm{M} 71$ transgenic mice. These data confirm that intrabulbar projection plasticity after naris block and reopening is a universal response and not restricted to a particular line of transgenic mice.

\section{Time course of change in P2 ORNs}

Since studies have shown that sensory deprivation can also affect the olfactory epithelium, we next examined the time course of

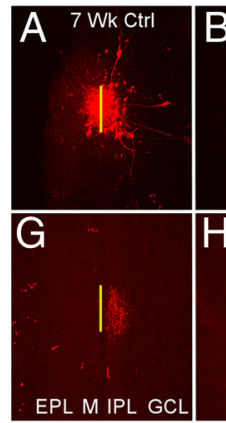

$\mathrm{Y}$
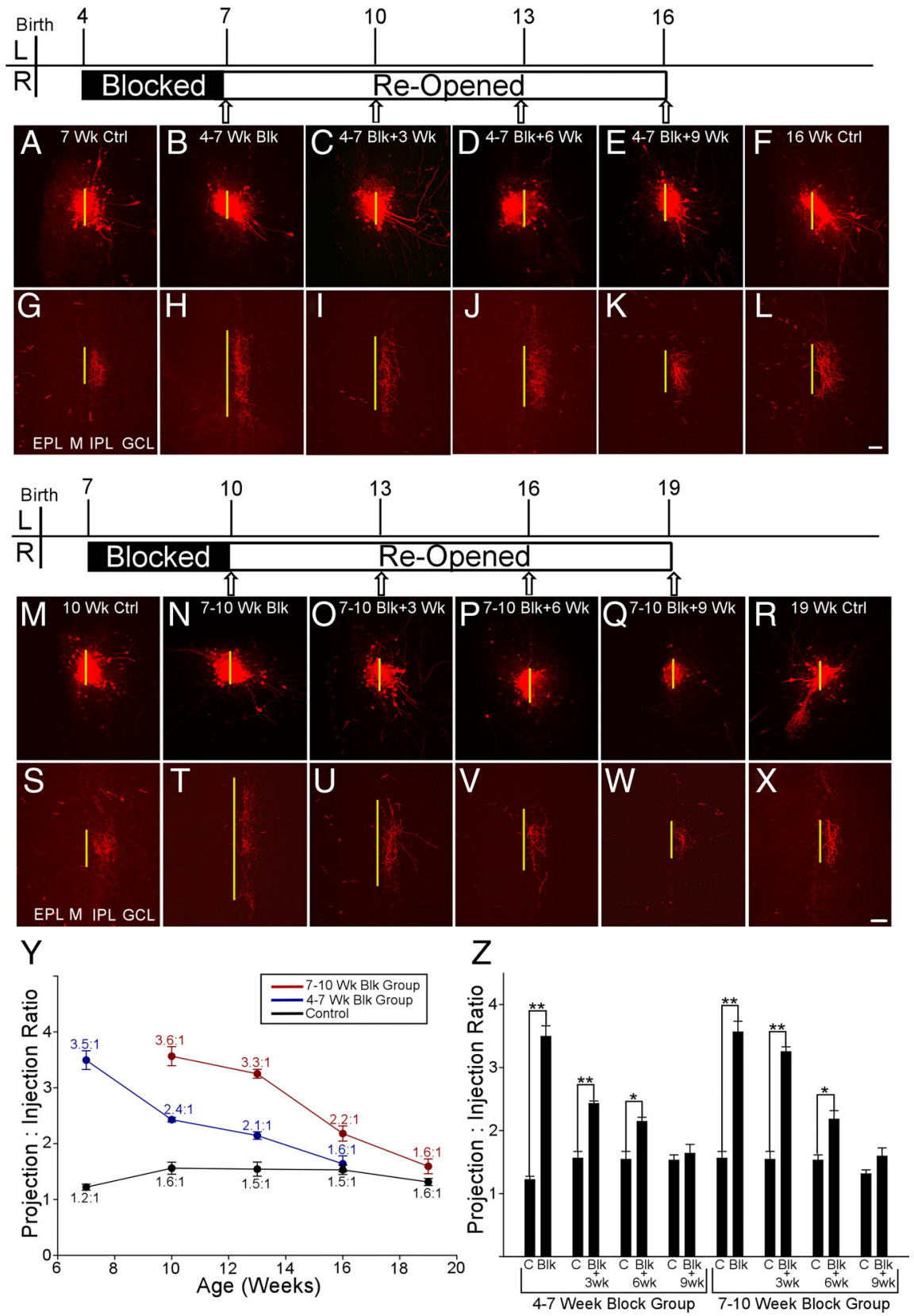

Figure 1. Confocal images of discrete TMR tracer injections and axonal projections after naris closure from 4 to 7 or 7 to 10 weeks. Time lines indicate weeks of naris closure and reopening, the time points when tracer injections were done (arrows), and the side of the nasal cavity that was blocked (the right side, R). $\boldsymbol{A}-\boldsymbol{L}$, TMR tracer injections $(\boldsymbol{A}-\boldsymbol{F})$ are shown in the dorsolateral $0 \mathrm{~B}$ of $\mathrm{rl} 7 \rightarrow \mathrm{M} 71$ mice with corresponding intrabulbar projection sites $(\boldsymbol{G}-\boldsymbol{L})$. Yellow lines represent the greatestlengths of injection and projection sites. $\boldsymbol{A}-\boldsymbol{L}$, Injection and projection series from juvenile mice following unilateral naris closure from 4 to 7 weeks of age $(\boldsymbol{B}, \boldsymbol{H})$, along with subsequent recovery periods of 3 weeks $(\boldsymbol{C}, \boldsymbol{I}), 6$ weeks $(\boldsymbol{D}, \boldsymbol{J})$, and 9 weeks $(\boldsymbol{E}, \boldsymbol{K})$ showing gradual refinement of the intrabulbar map. Control mice at 7 weeks $(\boldsymbol{A}, \boldsymbol{G})$ and 16 weeks $(\boldsymbol{F}, \boldsymbol{L})$ of age are included for comparison. $\boldsymbol{M}-\boldsymbol{X}$, Similar series in adult mice show tracer injections $(\boldsymbol{M}-\boldsymbol{R})$ and corresponding projection sites $(\boldsymbol{S}-\boldsymbol{X})$ of animals blocked from 7 to 10 weeks $(\boldsymbol{N}, \boldsymbol{T})$ and those followed by 3 weeks $(\boldsymbol{O}, \boldsymbol{U}), 6$ weeks $(\boldsymbol{P}, \boldsymbol{V})$ or 9 weeks $(\boldsymbol{Q}, \boldsymbol{W})$ of recovery as well as 10 -week $(\boldsymbol{M}, \boldsymbol{S})$ and 19 -week $(\boldsymbol{R}, \boldsymbol{X})$ controls, showing that the expansion and gradual re-refinement of intrabulbar projections does not have a critical period. Graphs illustrate mean projection to injection ratios ( \pm SEM) and the trend of projection broadening and refinement observed in both age groups $(\boldsymbol{Y})$ along with the significant differences observed after the $4-7$ and $7-10$ week blocks $\left({ }^{*} p<0.001, t=15.40\right.$ and 12.04 , respectively) as compared to age matched controls (Z). Significant differences were also found after $4-7$ or $7-10$ weeks of naris closure with 3 weeks ( ${ }^{*} p<0.001, t=6.29$ and 10.23 , respectively) or 6 weeks $\left({ }^{*} p<0.001\right.$, $t=4.34$ and ${ }^{*} p<0.001, t=3.90$, respectively) but not 9 weeks ( $p=0.44, t=0.78$ and $p=0.16, t=1.44$, respectively) of recovery (Z). R, Right side; L, left side; $M$, mitral cell layer. Scale bars, $50 \mu \mathrm{m}$.

change occurring among OSNs after naris closure. Specifically, we quantified a subset of OSNs that express lac $Z$ under the control of the promoter for a single OR known as P2 using P2-IREStaulacZ mice (Mombaerts et al., 1996). LacZ-positive cells were 

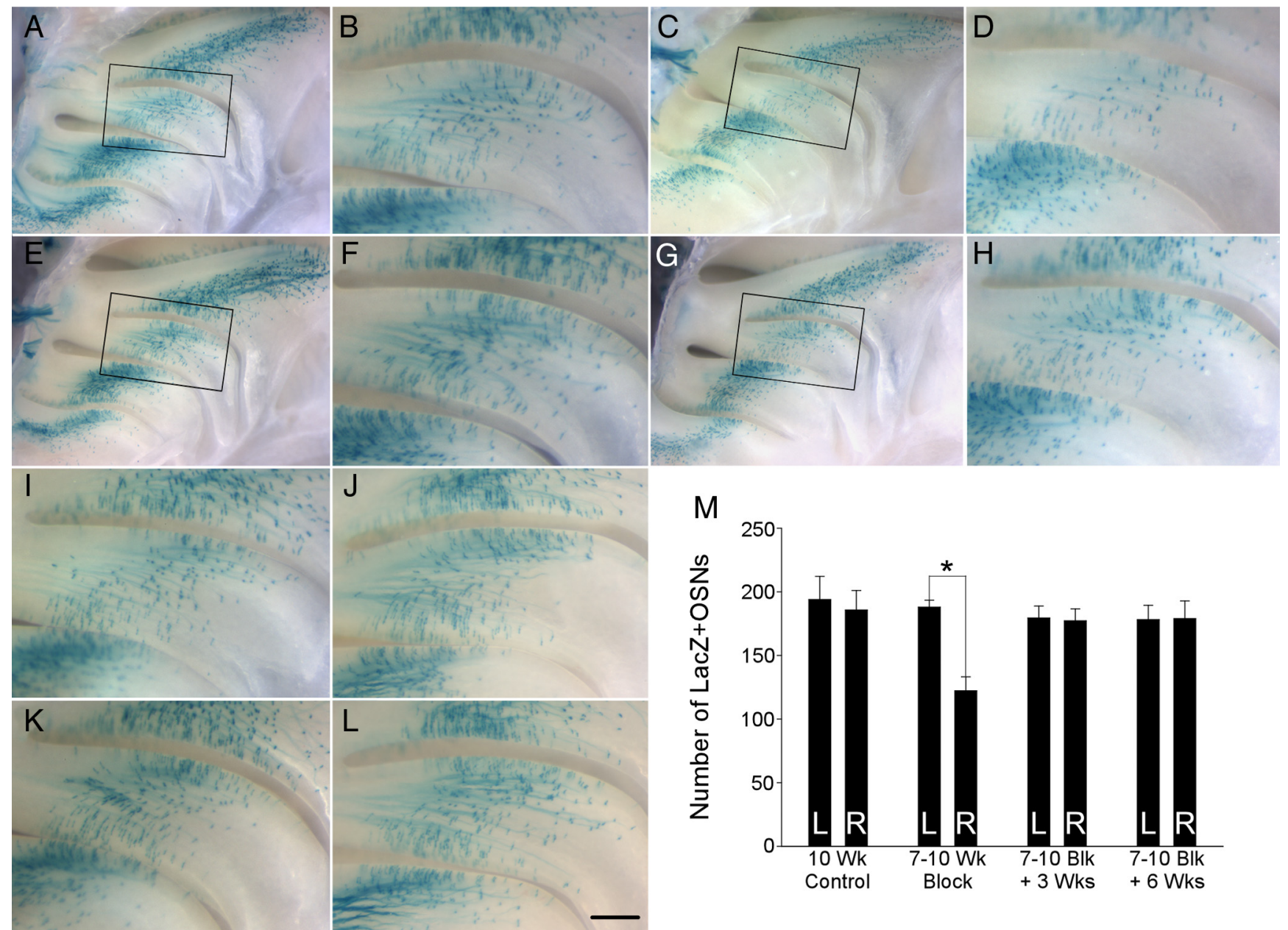

Figure 2. Photomicrographs of the lateral turbinates of P2-IRES-taulacZ mice. $A-L$, Mice served as controls $(A, B, E, F)$ or underwent reversible naris closure from 7 to 10 weeks $(C, D, G, H)$, or from 7 to 10 weeks with 3 weeks $(\boldsymbol{I}, \boldsymbol{K})$ or 6 weeks $(\boldsymbol{J}, \boldsymbol{L})$ of recovery. Lateral turbinates in the experimental side (right) of the nasal cavity are shown in $\boldsymbol{C}, \boldsymbol{D}, \boldsymbol{I}$, and $\boldsymbol{J}$, while contralateral control (left) turbinates are shown in $\mathbf{G}, \boldsymbol{H}, \boldsymbol{K}$, and $\boldsymbol{L}$. $\boldsymbol{M}$, Graph shows the average number of lacZ + cells in turbinate II ( $\pm S E M)$, revealing a significant reduction on the experimental side in the blocked group $\left({ }^{*} p<0.01 ; t=4.5\right)$ but no significant difference in cell number after naris block and reopening for 3 or 6 weeks or in controls. These results suggest that removing the block leads to a relatively rapid upregulation of Lac-Z+ cells on the previously occluded side. L, Left; R, right. Scale bar (in $L$ ): $A, C, E, G, 500 \mu \mathrm{m} ; \boldsymbol{B}, \mathbf{D}, \boldsymbol{F}, \boldsymbol{H}, \boldsymbol{I}-\mathbf{L}, 350 \mu \mathrm{m}$.

counted along lateral turbinate II in P2-IRES-taulacZ mice that underwent naris closure from 7 to 10 weeks of age followed by 3 or 6 weeks of normal olfactory stimulation and compared to age-matched controls (Fig. $2 A-L$ ). Interestingly, there was a substantial reduction in the number of $l a c Z+$ cells in the experimental side of the nasal cavity after the $7-10$ week block $\left({ }^{*} p<0.005\right.$; $t=5.39)$, but not after the block and 3 weeks of recovery $(p=$ $0.87 ; t=0.17$ ) or 6 weeks of recovery $(p=0.97 ; t=-0.5)$ (Fig. $2 M)$. These results show that reduced levels of odorant-induced activity cause a striking decrease in numbers of mature P2 OSNs on the deprived side of the nasal cavity. Furthermore, these data indicate that restoring normal afferent activity leads to a relatively rapid recovery in numbers of mature P2 OSNs.

\section{Alterations in the glomerular map}

The sharp distinction in the recovery time line between the intrabulbar map and P2 OSN numbers raised the question of how quickly changes occur in the glomerular layer follow alterations in odorant-induced activity. Since OSNs send axons to appropriate glomeruli to form the glomerular map, we hypothesized that any distortion in the glomerular map may also be corrected relatively quickly. To test this, we examined the P2-LacZ lateral glomeruli associated with the P2-IRES-taulac $Z$ mice that under- went (1) naris closure from 7 to 10 weeks, (2) naris closure followed by reopening for either 3 or 6 weeks, and (3) controls (Fig. $3 A-H)$. Volume measurements of immunolabeled P2-LacZ lateral glomeruli were collected in both the experimental and contralateral OBs and within animal differences were compared to those of controls, revealing a significant, average $55.7 \%$ reduction in P2 glomerular volume after naris closure from 7 to 10 weeks $\left({ }^{*} p<0.001 ; t=-6.0\right)$ (Fig. 3I). Interestingly, in as little as 3 weeks after plug removal, there was no significant difference in the volumes of P2 lateral glomeruli in experimental and contralateral OBs compared to controls ( $p=0.63 ; t=0.52$ ). In addition, no left-right differences were observed in P2 glomerular volume following naris closure and 6 weeks of recovery or in controls (Fig. 3I). Thus, the alterations in P2 lateral glomerular volume corresponded to the changes in numbers of X-galcontaining cells observed along lateral turbinate II. Volumes of medial P2 glomeruli were also measured and a similar trend emerged (see supplemental Fig. 2, available at www.jneurosci.org as supplemental material). In summary, the time course of recovery in the $\mathrm{OE}$ and within the first synaptic relay of the $\mathrm{OB}$, the glomerular layer, occurs much more rapidly than the changes seen among intrabulbar map projections. 


\section{Changes in intrabulbar projection complexity}

To determine whether the activitydependent changes in the spread of intrabulbar projections are also reflected in the structure of the projection tuft, we reconstructed the axonal arbors produced by tracer injections of similar sizes in mice blocked from 7 to 10 weeks with recovery for 0,3 , or 6 weeks, and compared their complexity to control mice (Fig. 4A-D). We quantified the numbers of branch points across the full axonal tuft to determine branch point density and analyzed the data with a one-way ANOVA that showed significant differences across groups $\left(F_{(3,12)}=11.77 ;{ }^{\star} p<0.005\right)$. Post hoc tests using the Holm-Sidak method that compared these measurements in experimental and control mice revealed a reduction in the branch point densities of mice that underwent naris closure from 7 to 10 weeks and in mice that experienced naris block from 7 to 10 weeks followed by 3 weeks of recovery $\left({ }^{*} p<0.05, t=5.54\right.$ and ${ }^{\star} p<0.05, t=4.31$, respectively) (see Table 1). Branch point density in narisclosed animals that experienced 6 weeks of recovery was not significantly different from controls. These data indicate that the character of intrabulbar axonal tufts changes in response to alterations in odorant-induced activity, suggesting that postsynaptic connections may also be involved in modulating intrabulbar projection specificity.
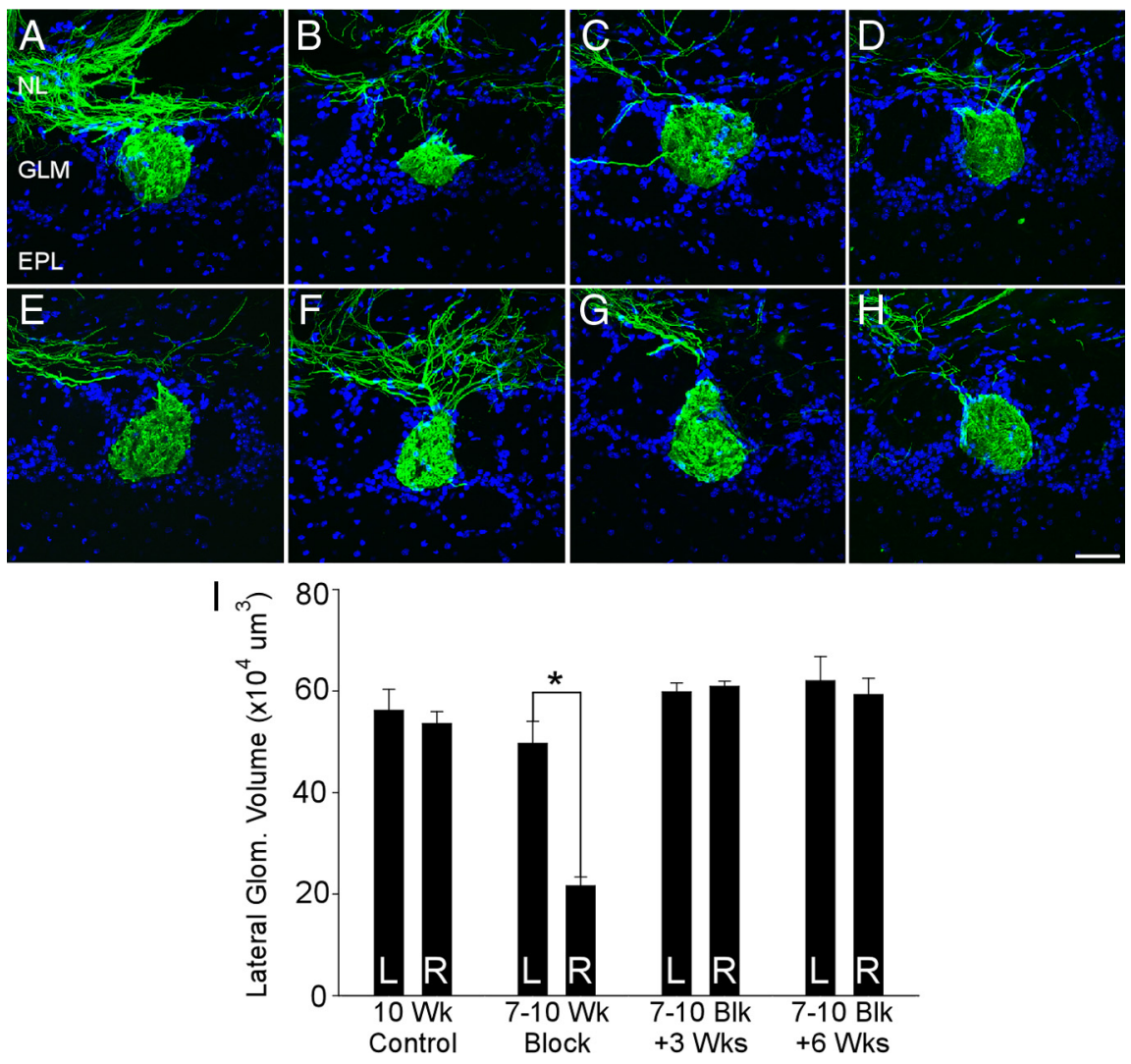

Figure 3. Confocal images of $\beta$-gal-ir lateral glomeruli in P2-IRES-taulacZ mice. $\boldsymbol{B}, \boldsymbol{F}$, Images of lateral P2 $\beta$-gal-ir glomeruli are shown (green) with a DAPI counterstain (blue) and illustrate an average $55.7 \%$ difference in glomerular volume found in mice that experienced reversible naris closure from 7 to 10 weeks of age $(n=4) . \boldsymbol{A}, \boldsymbol{C}, \boldsymbol{D}, \boldsymbol{E}, \boldsymbol{G}, \boldsymbol{H}$, This difference was not found in age-matched controls $(n=3)(\boldsymbol{A}, \boldsymbol{E})$ or in mice that underwent naris closure from 7 to 10 weeks followed by 3 weeks $(n=4)(\boldsymbol{C}$, $\boldsymbol{G})$ or 6 weeks $(n=3)(\boldsymbol{D}, \boldsymbol{H})$ of normal olfactory stimulation. $\boldsymbol{I}$, Graph shows average P2 glomerular volume measurements ( \pm SEM) and reveals a significant reduction on the experimental (right) side following naris closure from 7 to 10 weeks $\left({ }^{*} p<\right.$ $0.001 ; t=-6.0$ ), but no left-right differences after the block and 3 or 6 weeks of recovery. L, Left; R, right; NL, nerve layer; GLM, glomerular layer; Glom., glomerular. Scale bar, $50 \mu \mathrm{m}$.

\section{Alterations in the density of a granule cell synapse marker}

When odorant-induced activity is blocked, fewer newborn cells in the granule cell layer (GCL) survive and a greater number of granule cells die (Frazier-Cierpial and Brunjes, 1989b; Fiske and Brunjes, 2001; Saghatelyan et al., 2005). Interestingly, when activity levels are restored, the volume of the GCL and the levels of cell death in this layer return to normal (Cummings et al., 1997; Fiske and Brunjes, 2001). Since granule cells are the postsynaptic target neurons of intrabulbar projections (Liu and Shipley, 1994), we sought to determine whether activity-dependent changes in granule cell contacts correlate with our observed intrabulbar changes. We used immunohistochemistry to examine the density of a granule cell synapse marker, synaptoporin (Whitman and Greer, 2007), in both the EPL and IPL. Our data analysis demonstrated a significant effect across treatment groups (one-way ANOVA $\left.F_{(3,11)}=12.45, p<0.05\right)$ for synaptoporin-ir puncta densities within the IPL, and post hoc tests using the Holm-Sidak method revealed that activity deprivation from 7 to 10 weeks of age caused a significant decrease in the density of synaptoporinpositive puncta compared to control levels $\left({ }^{\star} p<0.05 ; t=4.51\right)$ (Fig. 5A, $B, E, F, I)$. By contrast, the density of synaptoporin-ir puncta within the EPL was not significantly affected $\left(F_{(3,11)}=\right.$ $1.03, p=0.43)$. In addition, our data showed a significant reduction in the density of synaptoporin-ir puncta within the IPL of animals that were blocked and allowed to recover for 3 weeks ${ }^{\star} p<0.05 ; t=3.51$ ) (Fig. $\left.5 C, G, I\right)$. However, after activity restoration for 6 weeks, the synaptoporin-positive puncta density returned to control levels in the IPL (Fig. 5D,H,I). This gradual recovery in synaptoporin density parallels intrabulbar projection restoration and suggests that granule cell contacts may play a role in regulating their plasticity.

\section{Discussion}

\section{Activity-dependent recovery of the intrabulbar map}

Our results demonstrate that both reduction and restoration of odorant-induced activity through reversible naris closure cause anatomical changes in the specificity of $\mathrm{OB}$ intrabulbar projections. The broadening and subsequent refinement of intrabulbar projections in response to changes in afferent activity during adulthood illustrates that neuronal circuitry within the olfactory system remains extremely dynamic. While this unique degree of plasticity has been implicated in studies that observed broad changes after naris closure, including decreases in epithelial thickness, OSN proliferation, interneuron survival, OB size, and expression of biochemical markers, and increased cell death (Farbman et al., 1988; Frazier-Cierpial and Brunjes, 1989a; Baker et al., 1993; Brunjes, 1994; Cummings and Brunjes, 1994; Cho et al., 1996; Fiske and Brunjes, 2001; Saghatelyan et al., 2005), our 

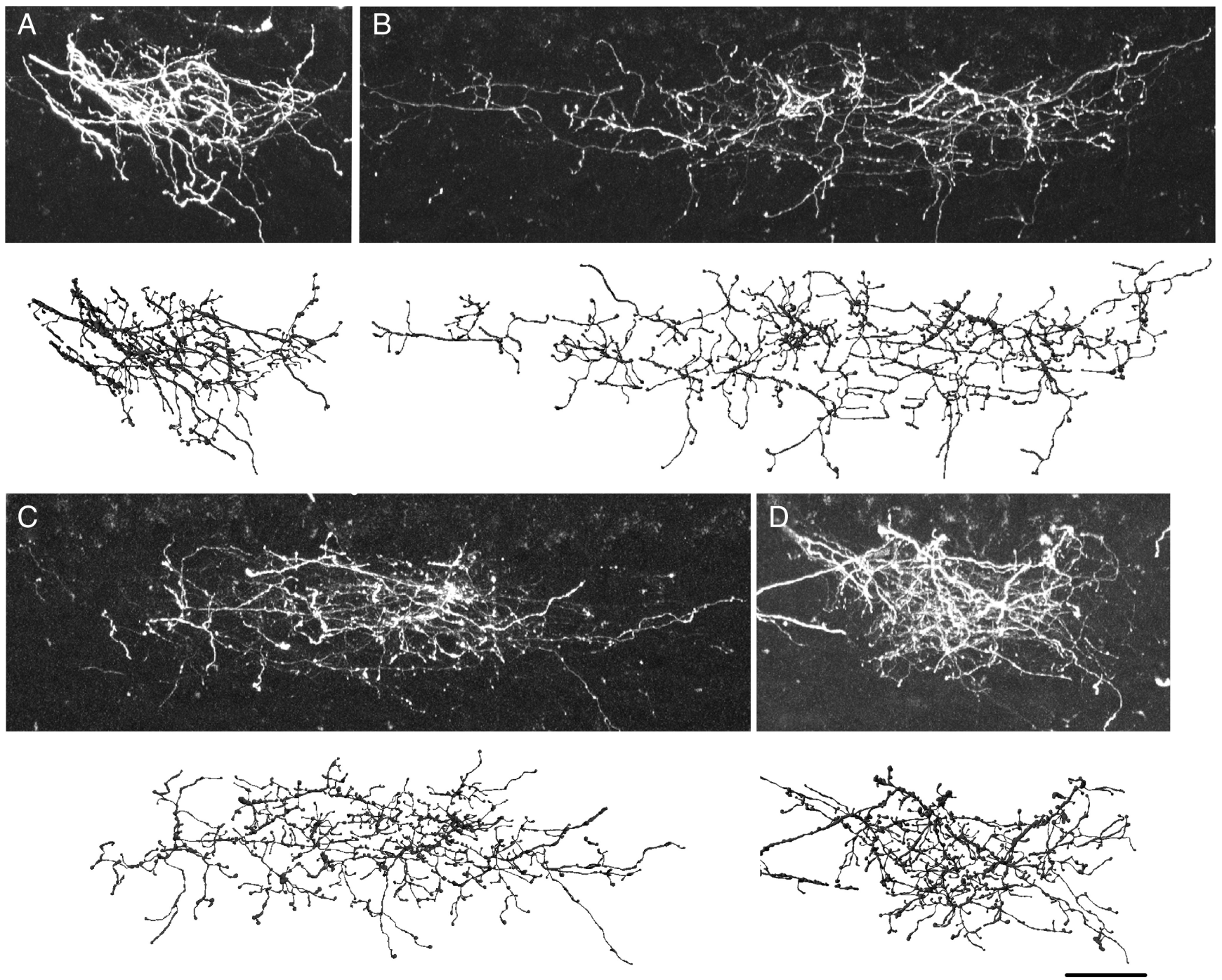

Figure 4. Three-dimensional reconstructions of intrabulbar axonal tufts. Flattened images of confocal stacks of intrabulbar axonal projection sites are shown with corresponding $3 D$ reconstructions below in each case. $\boldsymbol{A}-\boldsymbol{D}$, Representative confocal images and reconstructions are shown from 10-week-old controls $(\boldsymbol{A})$, mice that experienced naris closure from 7 to 10 weeks $(\boldsymbol{B})$, and mice that underwent naris block followed by 3 weeks $(\boldsymbol{C})$ or 6 weeks $(\boldsymbol{D})$ of normal levels of afferent activity. Scale bar, $50 \mu \mathrm{m}$.

Table 1. Averaged process length and branch point measurements of reconstructed intrabulbar projection sites

\begin{tabular}{lllll}
\hline Group & $n$ & $\begin{array}{l}\text { Average total filament } \\
\text { length } \pm \text { SEM }(\mu \mathrm{m})\end{array}$ & $\begin{array}{l}\text { Average no. } \\
\text { of branch } \\
\text { pts } \pm \text { SEM }\end{array}$ & $\begin{array}{l}\text { Branch pts/total } \\
\text { filament length } \\
(\mu \mathrm{m})\end{array}$ \\
\hline Controls & 3 & $6866 \pm 458$ & $560 \pm 57$ & 0.082 \\
$7-10$ wk blk & 4 & $9098 \pm 468$ & $515 \pm 22$ & $0.057^{*}$ \\
Blk +3 wks & 3 & $8497 \pm 301$ & $517 \pm 7$ & $0.061^{*}$ \\
Blk +6 wks & 3 & $6703 \pm 852$ & $478 \pm 72$ & 0.071
\end{tabular}

The table shows numbers of animals per group with total axonal filament length ( \pm SEM), average number (no.) of branch points (pts) ( \pm SEM), and the resulting average number of branch points per total filament length. Significant differences were observed in these branch point density values in $7-10$ week (wk) blocked (blk) mice ${ }^{*} p<$ $0.05, t=5.54)$ and in mice blocked from $7-10$ weeks followed by 3 weeks of recovery $\left({ }^{*} p<0.05, t=4.31\right)$ compared to controls. No significant difference emerged when branch point densities of mice blocked from 7-10 weeks plus 6 weeks of recovery were compared to controls.

study presents clear evidence of this ongoing plasticity within a specific circuit.

Previous experiments using reversible olfactory deprivation also revealed that removal of the naris block following a period of naris closure resulted in increased neuronal survival and volumetric recovery of the layers within the ipsilateral $\mathrm{OB}$
(Cummings et al., 1997). Similarly, research on olfactory bulb regeneration suggests that activity can lead to substantial increases in the integration of new neurons into existing OB circuitry (Rochefort et al., 2002; Alonso et al., 2006). These findings along with other work highlight the presence of long-term developmental changes and/or plasticity within the OE and OB, likely associated with regeneration in both regions (Weiler and Farbman, 1997; Lee et al., 2009; Zou et al., 2009). Our current findings demonstrate that reversible naris closure, initiated for 3 weeks either before or after the intrabulbar map matures, causes a spread in intrabulbar axons similar to those observed after permanent naris closure (Marks et al., 2006). Remarkably, we show that by reinstating normal levels of olfactory stimulation the intrabulbar projections can gradually refine and recover their specificity (Fig. 6). This suggests that the changes incurred by the olfactory bulb during sensory deprivation are not permanent but can be reversed. Thus, in addition to the general increases in neuronal survival and $\mathrm{OB}$ volume reported previously (Cummings et al., 1997), odorant-induced activity can also restore the specificity of the axonal connections that make up the intrabulbar map. 

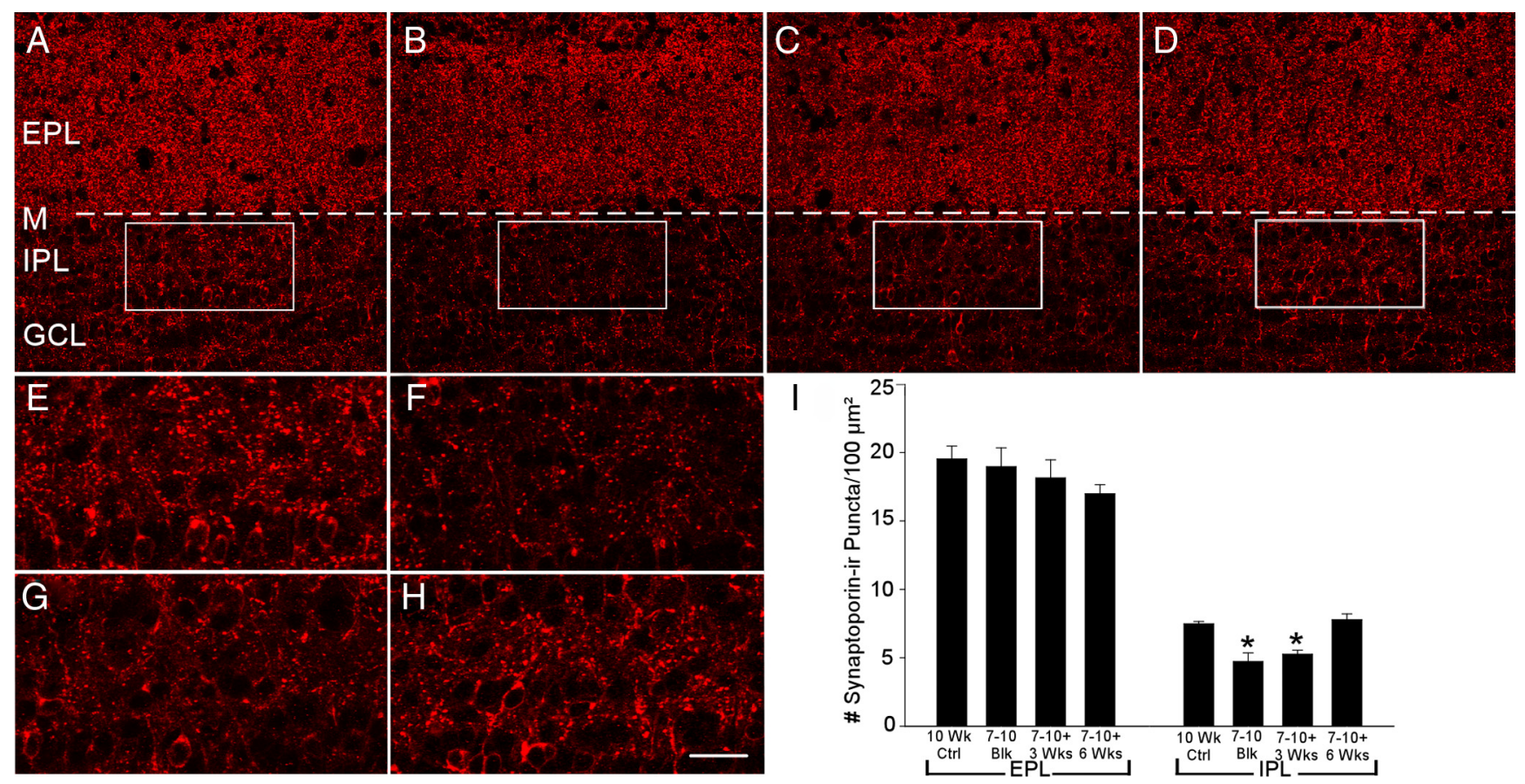

Figure 5. Activity-dependent changes in synaptoporin-ir puncta density within the EPL and IPL. $A-H$, Confocal images of synaptoporin immunostaining in $0 B$ sections from 10 week control mice $(\boldsymbol{A}, \boldsymbol{E})$, mice that underwent naris closure from 7 to 10 weeks $(\boldsymbol{B}, \boldsymbol{F})$, and mice that experienced naris closure followed by 3 weeks $(\boldsymbol{C}, \boldsymbol{G})$ or 6 weeks $(\boldsymbol{D}, \boldsymbol{H})$ of recovery. The white dotted line in $\boldsymbol{A}-\boldsymbol{D}$ delineates the mitral cell layer. Boxed regions of the IPL of $\boldsymbol{A}-\boldsymbol{D}$ are shown in higher magnification in $\boldsymbol{E}-\boldsymbol{H}$. $\boldsymbol{I}$, Graph shows the quantification of synaptoporin-ir puncta densities in the EPL and IPL. Naris closure led to a decrease in the density of synaptoporin-ir puncta in the IPL $\left({ }^{*} p<0.05 ; t=4.51\right)$, and this decrease persisted in mice that underwent olfactory deprivation followed by 3 weeks of recovery $\left({ }^{*} p<0.05 ; t=3.51\right)$, but not in the group that recovered for 6 weeks. In addition, no significant differences were observed in synaptoporin-ir puncta densities within the EPL. Scale bar (in $\boldsymbol{H}$ ): $\boldsymbol{A}-\boldsymbol{D}, 50 \mu \mathrm{m} ; \boldsymbol{E}-\boldsymbol{H}, 30 \mu \mathrm{m}$.

\section{Rapid return in OSNs and the glomerular map}

Given that intrabulbar map recovery is contingent upon olfactory sensory input, we examined activity-dependent changes in the olfactory epithelium by following P2-OSNs and their associate glomeruli. We reasoned that if olfactory input is required for intrabulbar map recovery, then it may be necessary for OSN changes to recover before the restoration of intrabulbar projection specificity, which is indeed what we found. We noted a $\sim 35 \%$ reduction in the number of $\mathrm{P} 2$-lacZ-expressing neurons after a 3 week period of naris occlusion which recovered to control levels within 3 weeks of removing the naris block (Fig. 2). While the mechanism underlying these epithelial changes is unclear, studies have shown that odorant deprivation beginning shortly after birth causes a reduction in neurogenesis and a decrease in epithelial thickness (Farbman et al., 1988; Cummings and Brunjes, 1994), suggesting that changes in OSN turnover are likely involved. In addition, factors such as a variation in air flow (Scott-Johnson et al., 2000) or mucus consistency (Getchell et al., 1987; Persaud et al., 1987) that result from the naris occlusion may also contribute to the epithelial fluctuations we observe.

At the glomerular level, our findings further demonstrate the rapid recovery of OSNs in response to renewed odorant-induced activity. Studies have shown that the glomerular map undergoes a period of activity-dependent postnatal refinement during which heterogeneous and ectopic glomeruli mature into more typical arrangements of isofunctional glomerular pairs (Treloar et al., 2002; Zou et al., 2004). Experience also appears to play a role in this maturation since odorant conditioning has been shown to enhance glomerular refinement (Kerr and Belluscio, 2006), and olfactory-dependent associative learning can increase the number of specific OSNs and associated glomerular size (Jones et al., 2008). In this study, we observed that $\mathrm{P} 2$ glomeruli undergo a reduction and subsequent restoration in volume that directly parallels the P2-OSN changes in the epithelium. Interestingly, P2 glomeruli remained intact and their position did not appear to change as a result of odorant deprivation, suggesting that glomerular location and composition may be relatively stable once the map matures.

\section{Postsynaptic changes associated with intrabulbar map plasticity}

We show that olfactory sensory input is clearly necessary to drive the refinement and restoration of intrabulbar projections. However, the remodeling process itself is very likely dependent upon postsynaptic factors as well. For example, the elements necessary for axonal reorganization in the IPL depend in part upon the destabilization, elimination, and/or addition of synaptic contacts. At a circuit level, intrabulbar projections form a communication link between two regenerating populations of neurons: primary OSNs within the glomerulus and granule cells on the opposite side of the bulb through synaptic contacts in the IPL. Thus, synaptic fluctuations within the IPL are a separate and somewhat complementary indicator of the plasticity that we observe directly through labeling intrabulbar axons, first revealing their ability to expand and contract; and second demonstrating a change in their complexity as revealed by our $3 \mathrm{D}$ reconstructions. In addition, we demonstrate through synaptoporin staining that activity deprivation produced a marked decrease in synaptic density within the IPL that returns to control levels following $\sim 6$ weeks of activity restoration (Fig. 5). These data are consistent with studies showing that blocking olfactory sensory input causes a decrease in the number of $\mathrm{OB}$ granule cells (Fiske and Brunjes, 2001), the postsynaptic partners of intrabulbar projections, sug- 

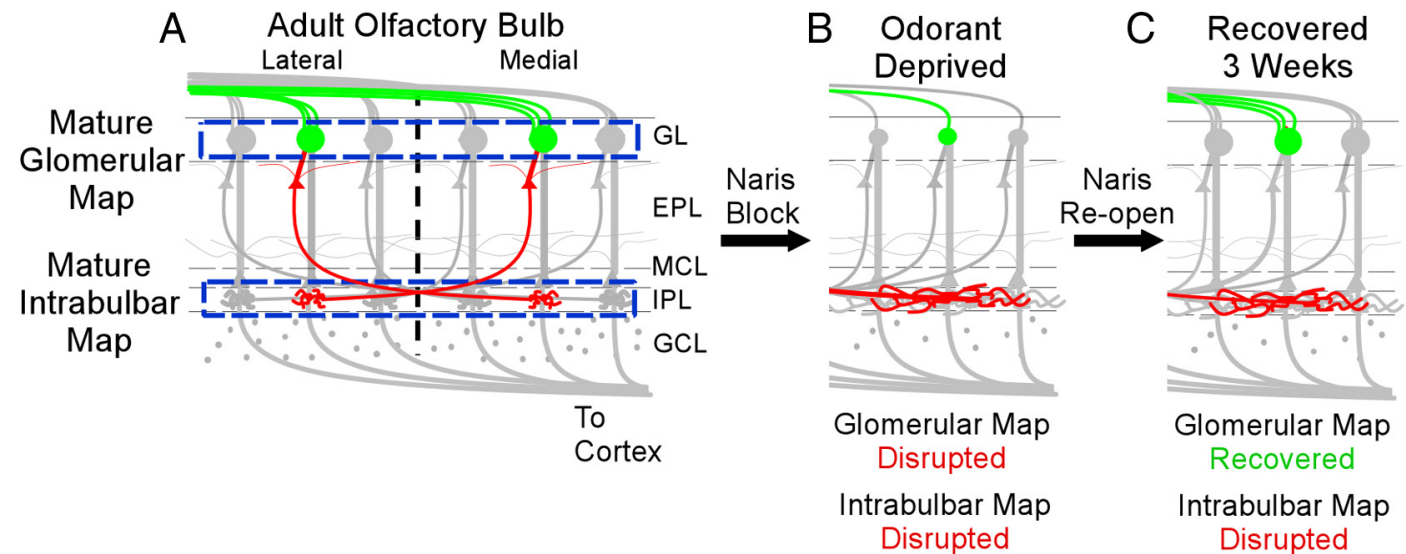

D Recovered 6-9 Weeks

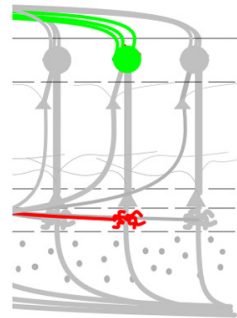

Glomerular Map

Recovered

Intrabulbar Map

Recovered

Figure 6. Model of intrabulbar map plasticity. $A$, In the adult OB, isofunctional glomeruli (shown in green) are connected by STC axons (shown in red) that reciprocally project to the region of the IPL located below the "partner" glomerulus. $\boldsymbol{B}$, If odorant-induced activity is blocked by naris closure, glomerular size becomes reduced and intrabulbar projections broaden. $\boldsymbol{C}$, Reopening the naris for 3 weeks results in the recovery of glomerular volume; however, the intrabulbar projections remain broad. $\boldsymbol{D}$, Reopening the naris and recovery for $\sim 6-9$ weeks allows the intrabulbar map to return to control levels of precision.

gesting that intrabulbar projection plasticity may be partially linked to granule cell turnover.

\section{The multiple maps of the olfactory bulb are functionally linked}

Most sensory systems undergo periods during their development when rudimentary connections become refined and can be shaped by the stimulus environment. For example, in both the visual and somatosensory systems, manipulating afferent activity during certain sensitive periods alters the arrangement of cortical representations (Hubel and Wiesel, 1970; Belford and Killackey, 1980; Fox, 1992). Although these systems have traditionally been characterized as lacking plasticity beyond these early critical windows, recent evidence suggests that adult plasticity may exist to some degree (Trachtenberg et al., 2002; De Paola et al., 2006; He et al., 2007). By comparison, the olfactory system maintains a continuous level of plasticity and regeneration both at the periphery in the $\mathrm{OE}$, and at central levels within the bulb. While the purpose of this plasticity is unclear, nowhere is it more obvious than in the activity-dependent changes presented in the intrabulbar map. We propose that intrabulbar projections are necessary to coordinate the communication between the two mirrorsymmetric glomerular maps, which in turn work together to generate specific patterns of synchronous activity that we believe are necessary for cortical integration of signals. In a previous study, we show that intrabulbar projecting neurons can modulate the firing of mitral cells through a timing-based gating mechanism (Zhou and Belluscio, 2008). Thus, given the anatomical specificity of the intrabulbar map, this mechanism could be used to selectively adjust the timing of mitral cell activity between pairs of isofunctional glomeruli. This in turn could be used to produce specific synchronous patterns of activity that may be interpreted within the piriform cortex to identify specific odorants.

Our results suggest that the specificity of intrabulbar projections depends upon the integrity of the sensory epithelium and glomerular map, since refinement among intrabulbar axons occurred only after OSN numbers and glomerular volume returned to normal. Indeed, the recovery of intrabulbar projection specificity occurs between 6 and 9 weeks following block removal (see Fig. 6), suggesting that this return to precision may depend upon multiple factors. Olfactory bulb granule cells, the postsynaptic targets of intrabulbar axons, may also play a role in modulating intrabulbar plasticity, possibly facilitating circuit dynamics sim- ply through their regenerative capacity. While the precise functional interactions between the many cell types of the $\mathrm{OB}$ warrant further study, we propose that the intrabulbar map derives its organization from distinct glomerular activity patterns that are transmitted through the odor column circuitry. Activation timing could then be used to stabilize or reinforce intrabulbar connections between isofunctional odor columns while destabilizing inappropriate connections to allow for axonal pruning. In addition, molecular factors that change with altered activity levels could also modulate the extension or retraction of axon terminals. Although specific mechanisms for such a process have yet to be determined, our results clearly indicate that sensory input reflected in the glomerular map ultimately drives the organizational maintenance and restoration of the intrabulbar map.

\section{References}

Alonso M, Viollet C, Gabellec MM, Meas-Yedid V, Olivo-Marin JC, Lledo PM (2006) Olfactory discrimination learning increases the survival of adult-born neurons in the olfactory bulb. J Neurosci 26:10508-10513.

Baker H, Morel K, Stone DM, Maruniak JA (1993) Adult naris closure profoundly reduces tyrosine hydroxylase expression in mouse olfactory bulb. Brain Res 614:109-116.

Belford GR, Killackey HP (1980) The sensitive period in the development of the trigeminal system of the neonatal rat. J Comp Neurol 193:335-350.

Belluscio L, Lodovichi C, Feinstein P, Mombaerts P, Katz LC (2002) Odorant receptors instruct functional circuitry in the mouse olfactory bulb. Nature 419:296-300.

Bozza T, Feinstein P, Zheng C, Mombaerts P (2002) Odorant receptor expression defines functional units in the mouse olfactory system. J Neurosci 22:3033-3043.

Brunjes PC (1994) Unilateral naris closure and olfactory system development. Brain Res Brain Res Rev 19:146-160.

Brunjes PC, Smith-Crafts LK, McCarty R (1985) Unilateral odor deprivation: effects on the development of olfactory bulb catecholamines and behavior. Brain Res 354:1-6.

Buck L, Axel R (1991) A novel multigene family may encode odorant receptors: a molecular basis for odor recognition. Cell 65:175-187.

Cho JY, Min N, Franzen L, Baker H (1996) Rapid down-regulation of tyrosine hydroxylase expression in the olfactory bulb of naris-occluded adult rats. J Comp Neurol 369:264-276.

Cummings DM, Belluscio L (2008) Charting plasticity in the regenerating maps of the mammalian olfactory bulb. Neuroscientist 14:251-263.

Cummings DM, Brunjes PC (1994) Changes in cell proliferation in the developing olfactory epithelium following neonatal unilateral naris occlusion. Exp Neurol 128:124-128.

Cummings DM, Brunjes PC (1997) The effects of variable periods of func- 
tional deprivation on olfactory bulb development in rats. Exp Neurol 148:360-366.

Cummings DM, Henning HE, Brunjes PC (1997) Olfactory bulb recovery after early sensory deprivation. J Neurosci 17:7433-7440.

Cummings DM, Emge DK, Small SL, Margolis FL (2000) Pattern of olfactory bulb innervation returns after recovery from reversible peripheral deafferentation. J Comp Neurol 421:362-373.

De Paola V, Holtmaat A, Knott G, Song S, Wilbrecht L, Caroni P, Svoboda K (2006) Cell type-specific structural plasticity of axonal branches and boutons in the adult neocortex. Neuron 49:861-875.

Farbman AI, Brunjes PC, Rentfro L, Michas J, Ritz S (1988) The effect of unilateral naris occlusion on cell dynamics in the developing rat olfactory epithelium. J Neurosci 8:3290-3295.

Fiske BK, Brunjes PC (2001) Cell death in the developing and sensorydeprived rat olfactory bulb. J Comp Neurol 431:311-319.

Fox K (1992) A critical period for experience-dependent synaptic plasticity in rat barrel cortex. J Neurosci 12:1826-1838.

Frazier-Cierpial L, Brunjes PC (1989a) Early postnatal cellular proliferation and survival in the olfactory bulb and rostral migratory stream of normal and unilaterally odor-deprived rats. J Comp Neurol 289:481-492.

Frazier-Cierpial LL, Brunjes PC (1989b) Early postnatal differentiation of granule cell dendrites in the olfactory bulbs of normal and unilaterally odor-deprived rats. Brain Res Dev Brain Res 47:129-136.

Getchell ML, Zielinski B, DeSimone JA, Getchell TV (1987) Odorant stimulation of secretory and neural processes in the salamander olfactory mucosa. J Comp Physiol A 160:155-168.

He HY, Ray B, Dennis K, Quinlan EM (2007) Experience-dependent recovery of vision following chronic deprivation amblyopia. Nat Neurosci 10:1134-1136.

Hubel DH, Wiesel TN (1970) The period of susceptibility to the physiological effects of unilateral eye closure in kittens. J Physiol 206:419-436.

Jones SV, Choi DC, Davis M, Ressler KJ (2008) Learning-dependent structural plasticity in the adult olfactory pathway. J Neurosci 28:13106-13111.

Kerr MA, Belluscio L (2006) Olfactory experience accelerates glomerular refinement in the mammalian olfactory bulb. Nat Neurosci 9:484-486.

Lee AC, Tian H, Grosmaitre X, Ma M (2009) Expression patterns of odorant receptors and response properties of olfactory sensory neurons in aged mice. Chem Senses 34:695-703.

Liu WL, Shipley MT (1994) Intrabulbar associational system in the rat olfactory bulb comprises cholecystokinin-containing tufted cells that synapse onto the dendrites of GABAergic granule cells. J Comp Neurol 346:541-558.

Lodovichi C, Belluscio L, Katz LC (2003) Functional topography of connections linking mirror-symmetric maps in the mouse olfactory bulb. Neuron 38:265-276.

Marks CA, Cheng K, Cummings DM, Belluscio L (2006) Activitydependent plasticity in the olfactory intrabulbar map. J Neurosci 26:11257-11266.
Mombaerts P, Wang F, Dulac C, Chao SK, Nemes A, Mendelsohn M, Edmondson J, Axel R (1996) Visualizing an olfactory sensory map. Cell $87: 675-686$.

Nakatani H, Serizawa S, Nakajima M, Imai T, Sakano H (2003) Developmental elimination of ectopic projection sites for the transgenic OR gene that has lost zone specificity in the olfactory epithelium. Eur J Neurosci 18:2425-2432.

Persaud KC, DeSimone JA, Getchell ML, Heck GL, Getchell TV (1987) Ion transport across the frog olfactory mucosa: the basal and odorantstimulated states. Biochim Biophys Acta 902:65-79.

Ressler KJ, Sullivan SL, Buck LB (1994) Information coding in the olfactory system: evidence for a stereotyped and highly organized epitope map in the olfactory bulb. Cell 79:1245-1255.

Rochefort C, Gheusi G, Vincent JD, Lledo PM (2002) Enriched odor exposure increases the number of newborn neurons in the adult olfactory bulb and improves odor memory. J Neurosci 22:2679-2689.

Saghatelyan A, Roux P, Migliore M, Rochefort C, Desmaisons D, Charneau P, Shepherd GM, Lledo PM (2005) Activity-dependent adjustments of the inhibitory network in the olfactory bulb following early postnatal deprivation. Neuron 46:103-116.

Scott-Johnson PE, Blakley D, Scott JW (2000) Effects of air flow on rat electroolfactogram. Chem Senses 25:761-768.

Suh KS, Kim SY, Bae YC, Ronnett GV, Moon C (2006) Effects of unilateral naris occlusion on the olfactory epithelium of adult mice. Neuroreport 17:1139-1142.

Trachtenberg JT, Chen BE, Knott GW, Feng G, Sanes JR, Welker E, Svoboda $\mathrm{K}$ (2002) Long-term in vivo imaging of experience-dependent synaptic plasticity in adult cortex. Nature 420:788-794.

Treloar HB, Feinstein P, Mombaerts P, Greer CA (2002) Specificity of glomerular targeting by olfactory sensory axons. J Neurosci 22:2469-2477.

Vassar R, Chao SK, Sitcheran R, Nuñez JM, Vosshall LB, Axel R (1994) Topographic organization of sensory projections to the olfactory bulb. Cell 79:981-991.

Weiler E, Farbman AI (1997) Proliferation in the rat olfactory epithelium: age-dependent changes. J Neurosci 17:3610-3622.

Whitman MC, Greer CA (2007) Synaptic integration of adult-generated olfactory bulb granule cells: basal axodendritic centrifugal input precedes apical dendrodendritic local circuits. J Neurosci 27:9951-9961.

Zhou Z, Belluscio L (2008) Intrabulbar projecting external tufted cells mediate a timing-based mechanism that dynamically gates olfactory bulb output. J Neurosci 28:9920-9928.

Zhuang H, Matsunami H (2007) Synergism of accessory factors in functional expression of mammalian odorant receptors. J Biol Chem 282:15284-15293.

Zou DJ, Feinstein P, Rivers AL, Mathews GA, Kim A, Greer CA, Mombaerts P, Firestein S (2004) Postnatal refinement of peripheral olfactory projections. Science 304:1976-1979.

Zou DJ, Chesler A, Firestein S (2009) How the olfactory bulb got its glomeruli: a just so story? Nat Rev Neurosci 10:611-618. 